RESEARCHARTICLE

\title{
Improved Batting Performance through a Novel Resistance Training Program
}

\author{
David T Burke ${ }^{1 *}$, Tracie McCargo ${ }^{2}$, Regina Bell', Daniel P. Burke ${ }^{3}$ and Cleo D. Stafford \\ II \\ ${ }^{1}$ Department of Rehabilitation Medicine, Emory University School of Medicine, Atlanta, Emory \\ University, USA \\ ${ }^{2}$ Harvard Extension School, Harvard University, USA \\ ${ }^{3}$ Georgia College and State University, USA \\ ${ }^{4}$ Department of Orthopaedics, Emory University School of Medicine, USA
}

\begin{abstract}
Purpose: This paper investigates the effect of adding to the off-season training program an eastern medicine weightlifting program, Gravitational Wellness ${ }^{\circledR}(\mathrm{GW})$ on the subsequent season batting performance of a Division I baseball team.

Methods: This study used retrospective data collected on in-season batting performance statistics for 40 Division I baseball players during seasons 2001 through 2007. The GW intervention group consisted of 9 positional players who participated in both the 2007 and 2008 seasons and engaged in $\mathrm{GW}^{\circledast}$ training during the off season after 2007. At each $\mathrm{GW}^{\otimes}$ exercise session, the participant engaged in four separate exercises, all involving free weights using a barbell system. Change in batting performance from the 2007 and 2008 seasons was computed and compared to the year to year change in batting performance of the historic controls. The amount of weight lifted by the intervention group with each of the exercises was compared at weeks 1 and 10.

Results: Batting performance in home runs, batting average, and runs batted-in all improved in the intervention group, with all improvements greater than those found in the historic controls. This difference was significant in favor of $\mathrm{GW}^{\otimes}$ weight training in batting average $\left(F_{1,47}=11.2, p=0.002\right)$, but did not reach statistical significance for home runs $\left(F_{1,47}=\right.$ $0.011, p=0.916)$, or runs batted-in $\left(F_{1,47}=1.392, p=0.244\right)$.
\end{abstract}

Bell R, Burke DR, Stafford CD. Improved Batting Performance through a Novel Resistance Training Program. Physical Medicine and Rehabilitation. 2018;1(1):5.

Received: September 21, 2017 Accepted: December 28, 2017

Published: January 24, 2018

Copyright: $\odot 2018$ Burke et al. This is an open access article distributed under the terms of the Creative Commons Attribution License, which permits unrestricted use, distribution, and reproduction in any medium, provided the original author and source are credited.

Corresponding author: David T Burke, Department of Rehabilitation Medicine, Emory University Schoolof Medicine, USA

E-mail: dburke2@emory.edu

\section{Keywords}

Weight training, resistance training, gravitational wellness, batting performance

\section{Introduction}

Performance enhancement has long been a topic of interest for baseball athletes and coaches. When viewing competitive baseball from a scientific perspective, it is explosive and ballistic in nature. Success, particularly in batting, is thought to be largely dependent on power. ${ }^{1}$ Hoffman, in a review of professional baseball players found significant positive relations between lower-body power performance and home runs, total bases, and slugging percentage. ${ }^{2}$ Strength, power, and agility have also been found to correlate with subjective evaluation of batting and fielding performance ${ }^{3,4}$ Given the strong relationship between strength/power and baseball-specific performance, ${ }^{4,5}$ it is not surprising that the vast majority of conditioning programs in high-level baseball emphasize strength and power development. This manuscript investigates the relationship between pre-season training with the Gravitational Wellness ${ }^{\circledR}(\mathrm{GW})$ weightlifting system and the batting performance of a Division I baseball team.

In strength and conditioning research, there has been debate as to what are the most 
effective protocols to improve sport-specific performance in baseball players. Much of the current information is based on experimental models and anecdotal evidence. Ebben et al conducted a survey of MLB strength and conditioning coaches. ${ }^{6}$ Of the 30 teams, 21 responded and reported that they train in strength and power development off-season 3-5 days per week. ${ }^{6} 95 \%$ of the respondents stated that they use plyometric exercises, with $28 \%$ using Olympic-style lifts. ${ }^{6}$ Many of the coaches identified squat and/or lunge variations as very important exercises. ${ }^{6}$

Bat swing velocity is thought to be a key attribute to a good baseball hitter. ${ }^{7}$ Increased bat swing velocity is thought to be beneficial as it allows for decreased swing time, increased decision time, and increased batted ball velocity. ${ }^{8}$ In an evaluation of the kinematic differences between the baseball swing of hitters of varying skill levels, bat swing velocity was established as a key characteristic in identifying skill level and performance. ${ }^{9}$ Studies that focus on increasing a batter's swing velocity include Szymanski and colleagues, who reviewed factors contributing to increased bat velocity and found that individuals with the greatest strength and lean body mass had the greatest bat swing velocity. ${ }^{10}$ Szymanski et al also performed an anthropometric evaluation of high school baseball players and found a significant relationship between strength/power and linear bat velocity. ${ }^{11}$ Among those who compared these variables with performance during competition, there were no significant correlations between upper body strength, batting average, and strike rate. ${ }^{12}$

Despite this and other conditioning studies however, little data exists concerning the effects of changing certain parameters of the swing, and actual on-field performance during competition. Still others have focused on sport-specific practice and the development of complex pattern recognition, perceptual learning, attention and observational learning. Few studies have focused on specific training methods, and their effect on the outcome of specific training techniques. We expanded our literature search to include batting in cricket, as well as baseball, and found that in the research conducted, there is no significant correlations between upper body strength, batting average, and strike rate for both the 1-Day and T/20 matches in research conducted..$^{13}$

A vast majority of existing studies have evaluated the effects of training programs on sportspecific tests and simulations rather than on live, in-game performances. To the authors' knowledge there have been no studies detailing the relationship between specific off-season exercise programs and in-season game performance. This study reviewed the data gathered by a Division I baseball team that employed $\mathrm{GW}^{\circledR}$ as an adjunct to their off season training regimen. The $\mathrm{GW}^{\circledR}$ system was developed in Russia by Anatoly Samodoumov. This system uses rapidly progressive and heavy weights, lifted in short arcs using four basic movements. Previously published studies of $\mathrm{GW}^{\circledR}$ have shown the ability to load the musculoskeletal system with extremely high weights, with rapid weekly gains in weight loads and few injuries. ${ }^{14,15}$ Investigations of $\mathrm{GW}^{\circledR}$ have also demonstrated improvements in musculoskeletal symptoms and osteoporosis, as well as decreased thoracic/lumbar spine pain. ${ }^{16}$ However, no data have been published concerning the effect of this weightlifting technique on individual performance.

\section{Methods}

This study is a retrospective review of data collected in part by the training staff of a Division I male baseball team. Published batting performance data was reviewed for 40 individual players from 2001 to 2008 . Upon completion of the 2007 season, the training staff trialed the GW weightlifting program as an adjunct to the ongoing training of members of the baseball team.

To minimize the time needed for players to participate in $\mathrm{GW}^{\circledast}$ training, the weightlifting equipment was transported to the university in Atlanta, Georgia. Data were collected by the training staff during the weightlifting sessions (30-minutes, once per week) on the weight lifted at each of the four weightlifting stations, and the number of sessions that each team member attended.

At each $\mathrm{GW}^{\circledast}$ exercise session, the participant engaged in four separate exercises, all involving free weights using a barbell system (Figure 1). Weights were added until the instructor saw incorrect form, thus indicating that the maximal weight lifting capacity was being approached. Once the instructor noticed that the participant's form was faltering, the 
weights were reduced by $30 \%$, and the subject was asked to complete 3 additional repetitions as a cool down maneuver.

The belt lift (Figure 1A), involved the use of a barbell connected to a hoisting belt, which was attached at each end of the barbell to a connecting star. With the belt placed over the lower back, the participant was instructed to inhale, hold their breath, and to extend the knees from the starting angle of approximately $45^{\circ}$ to near full extension, avoiding the locking of the knees.

The hand lift (Figure 1B), used the barbell system positioned within a metal track, such that the starting point of a lift could occur with the patient's arms fully extended, and the knees bent at $45^{\circ}$. The subject was asked to keep the back aligned in an upward position, to lean away from the bar at $15^{\circ}$, and to retract the scapula. From this position the knees were extended, lifting the weights for a period of approximately 3 seconds.

The chest lift (Figure 1C), was performed from a lying position using weightlifting gloves. A chest press maneuver was then completed to full extension.

The final exercise, leg lift (Figure 1D), was performed from a lying position using a barbell system contained within upright metal tracks. The starting position was determined such that the knees were bent at $\mathrm{a}^{\circ} 5^{\circ}$ angle and with the participant instructed to extend the knees. The weights were lifted for a period of less than three seconds.

A

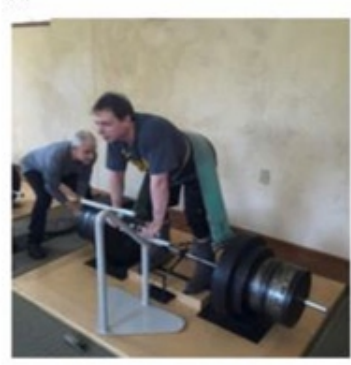

C

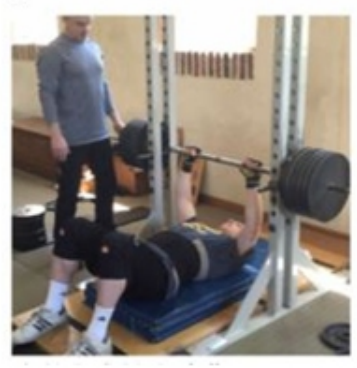

B

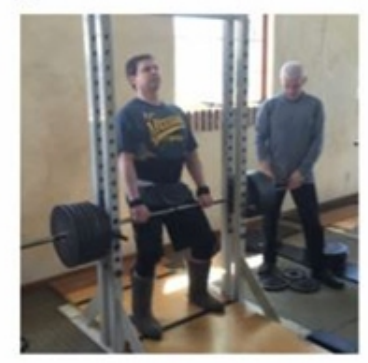

D

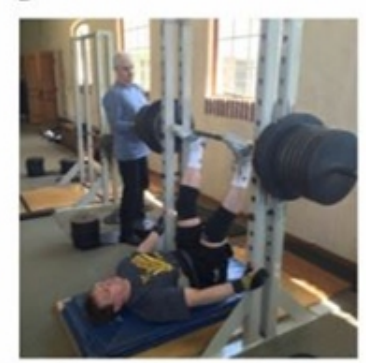

Figure 1. (A, B, C, D): Barbell system positioned according to the Gravitational Wellness ${ }^{\circledR}$ system

During the ensuing season, each player's batting average was determined by dividing the number of hits (successfully on base) by the number of times that the player was "at bat" $(A B)$. The home run (HR) statistic was determined by dividing the total number of home runs by the number of opportunities $A B$. Lastly, runs batted-in $(R B I)$ was assessed by dividing the total number of points scored during each $A B$ by the number of times $A B$. The results of these calculations were compared to the historic control group (CG).

\section{Results}

The primary outcome was the change in batting performance from one year to the following year. A comparison of means using one-way ANOVA and independent $t$-tests were conducted on the batting performance variables (both tests yielded the same results regarding significance). The average weight lifted was also analyzed. Calculations were performed using IBMs Statistical Package for the Social Sciences (SPSS).

Although the statistical trend for the 3 categories did not show true significance (Table 1), calculating the percentage of batting change did show that the GW intervention group had an overall clinical improvement (Figure 2). 
Table 1. Baseball Performance

\begin{tabular}{|l|r|r|r|r|r|r|}
\hline & & & & & \multicolumn{2}{|c|}{$\begin{array}{c}\text { 95\% Confidence } \\
\text { Interval }\end{array}$} \\
\cline { 2 - 7 } & & & & & \multicolumn{1}{c|}{ SIG } & \multicolumn{1}{c|}{ Upper } \\
\hline $\begin{array}{l}\text { Batting } \\
\text { Average }\end{array}$ & 0.48 & -1.086 & 0.140 & 0.039 & -0.12 & 0.04 \\
\hline Homerun* & 2.22 & -1.551 & 0.064 & 1.632 & -5.81 & 0.75 \\
\hline RBI & 1.75 & 0.003 & 0.499 & 6.46 & -12.98 & 13.01 \\
\hline
\end{tabular}

$p>0.05(p=0.064)$

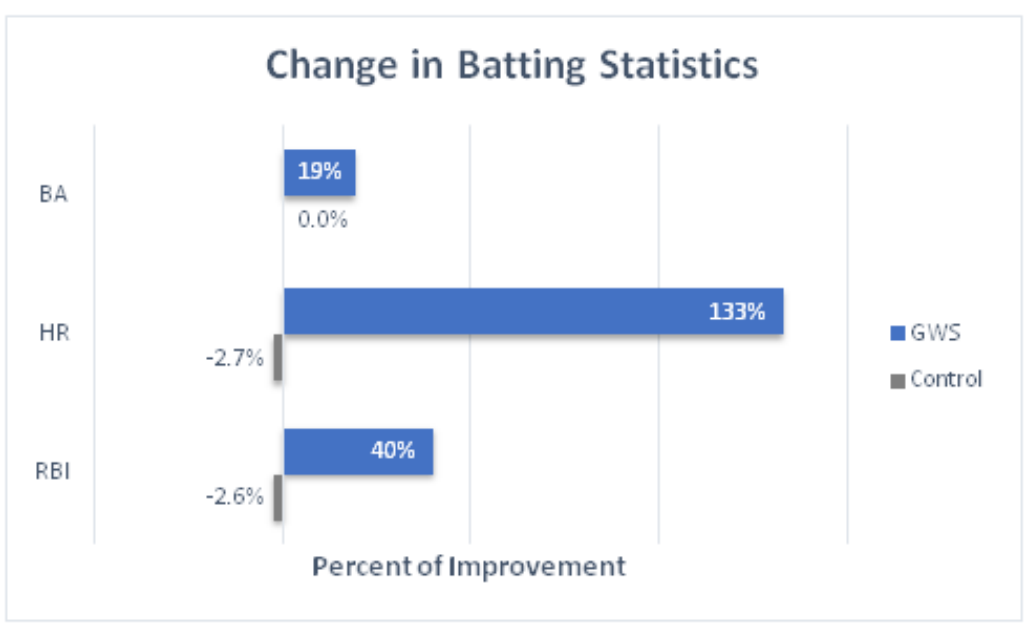

Figure 2. Change in Batting Statistics

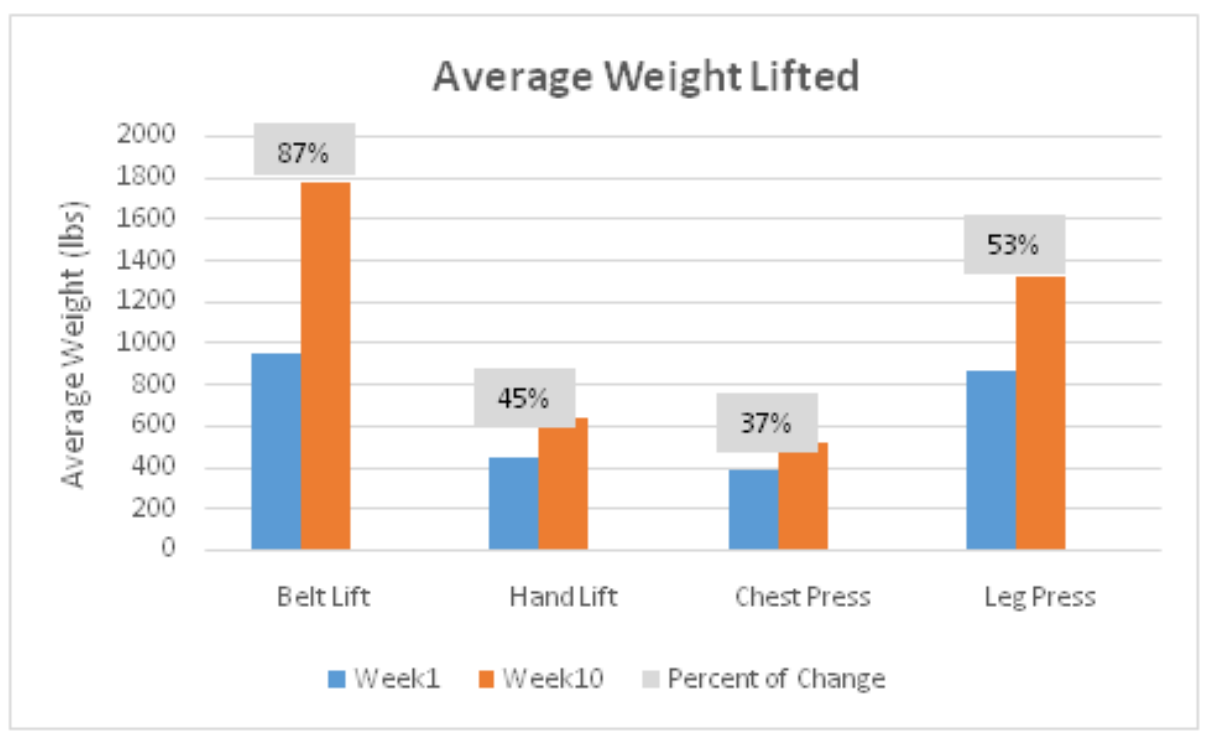

Figure 3. Average Weight Lifted

The intervention group gained the most improvement in HRs, followed by RBI and BA. The intervention group showed a $133 \%$ improvement in HR while the CG demonstrated a decline ($2.7 \%)$. The statistical trend was strongest in this group $(p=0.064)$. The intervention group had an overall $40 \%$ of improvement in RBIs and $19 \%$ in BA. The CG showed no improvement in BA and also declined in RBIs.

The secondary outcome was the average amount of weight lifted by the interventional group with each of the exercises (Figure 3). The mean of these was calculated for the first session, and the last session, with percent change visually depicted in Figure 3. For the belt lift, the average initial weight lifted was 951 pounds $(431.37 \mathrm{~kg})$. At the final session, the mean weight lifted was 1780 pounds ( $807.39 \mathrm{~kg}$ ), representing an $87 \%$ increase. For the hand lift, the mean initial lift was 441 pounds $(201.40 \mathrm{~kg})$, with a final mean lift of 645 pounds $(292.57 \mathrm{~kg})$, a $45 \%$ 
increase. For the chest press, average initial lift was 3782 pounds ( $173.27 \mathrm{~kg}$ ) with the final lift average of 523 pounds $(237.23 \mathrm{~kg})$, , a $37 \%$ increase. For the leg press, the initial average was 865 pounds $(392.36 \mathrm{~kg})$ with the final average of 1324 pounds $(600.56 \mathrm{~kg})$ pounds, a $54 \%$ increase.

\section{Discussion}

The primary aim of this study was to determine if an off-season high intensity weight lifting program could improve in-season batting performance. The data in this study demonstrate that the introduction of the $\mathrm{GW}^{\circledR}$ method in the off season conditioning of Division 1 baseball players correlated with an improvement in batting performance that was beyond that expected, based on historical data. To the authors' knowledge, this is one of the few studies to show objective positive in-game hitting performance directly correlated to an off-season conditioning program.

While much has been studied concerning the training regimens for improved hitting performance, few have undergone scientific scrutiny. In the literature concerning batting performance, work has been completed to determine the factors correlated with aspects of performance. These include upper extremity strength, velocity of bat swing, eye hand coordination, and visual tracking. None of these studies were designed to determine whether a change in the previously mentioned variables might produce a change in batting performance during competition. Further, we are aware of no studies that demonstrate that a process that is designed to increase bat velocity will result in an improvement in batting performance during competition.

The second aim of the study was to measure the increase in weight the athletes could manage during 30-minute weekly workout sessions over 10 weeks. The athletes experienced significant gains in the weight lifted by the end of the 10 weeks. The weights lifted by these athletes are notable, in that they far exceed numbers that are common among those who lift free weights.

The gains made by the intervention group are noteworthy, as previous studies have not found significant positive correlations between batting performance, particularly power performance (home runs, total bases, and runs batted-in), and weight lifted. Our study, using a training method that allows the lifting of greater weights is the first to correlate this change with a change in performance.

\section{Limitations}

One of the major limitations of the study was the use of a historical control group rather than a placebo-controlled group identified during the same season. This was necessary, as this study was initiated by the training staff as means to understand the effect of $\mathrm{GW}^{\circledR}$ on their team's performance, and was not initiated for scientific review.

A second limitation of the study was the low number of participants. Given that this was a pilot study undertaken by the training staff to determine the efficacy of a unique and local training program, there was no thought to randomize the participants at the onset of the trial.The data were carefully recorded during the training session so that the training staff could better evaluate the effect of the $\mathrm{GW}^{\circledR}$ program. When reviewing the data for this publication, we chose to include players who had sufficient playing time for meaningful performance averages. This reduced the number of individuals for whom we could calculate batting performance data. In total, these factors resulted in the inability to have robust statistical power to detect differences between the $\mathrm{GW}^{\circledR}$ and control groups.

Though the retrospective study contained only 9 players, the overall increase in their performance is certainly worthy of attention. Performance changes noted from year to year in the CG was essentially flat while those who engaged in $G^{\circledR}$ showed improvement. We suggest that a study with more participants and a randomized control, be conducted to better under stand the effect of this training technique.

To our knowledge, this is the first study that measures the performance changes in an elite baseball team after introducing one change in the training regimen. Given the nature of elite athletes, and performance, the fact that, after $\mathrm{GW}^{\circledR}$ training, every batting statistic measured improved greater than one the historic controls, these data are clinically compelling despite the low numbers and statistical power. 


\section{Conclusion}

This study of male athletes participating in a Division I baseball team found that those who participated in not only the team training program, but added the additional Gravitation Wellness ${ }^{\circledast}$ system, improved in batting performance as compared to historic controls. 


\section{References}

1. Rhea MR, BunkerD. Baseball-specific conditioning. Int J Sports Physiol Perform. 2009; 4(3): 402-407.

2. Hoffman JR, Vazquez J, Pichardo N, Tenenbaum G. Anthropometric and performance comparisons in professional baseball players. J Strength Cond Res. 2009; 23(8): 2173-2178.

3. Kohmura Y, Aoki K, Yoshigi H, et al. Development of a baseball-specific battery of tests and a testing protocol for college baseball players. J Strength Cond Res. 2008; 22(4): 1051-1058.

4. Mangine GT, Hoffman JR, Fragala MS, et al. Effect of age on anthropometric and physical performance measures in professional baseball players. J Strength Cond Res. 2013; 27(2):375-381.

5. Jones MT. Effect of compensatory acceleration training in combination with accommodating resistance on upper body strength in collegiate athletes. Open Access J Sports Med 2014; 5:183-189.

6. Ebben WP, MJ Hintz, Simenz CJ. Strength and conditioning practices of Major League Baseball strength and conditioning coaches. J Strength Cond Res. 2005; 19(3): 538-546.

7. Breen J. What makes a good hitter? J Health Phys Educ Rec. 1967. 38(4): 36-39.

8. DeRenne C.The Scientific Approach to Hitting. 2nd Ed. San Diego, CA: University Readers Custom Publishing; 2011.

9. Inkster B, Murphy A, Bower R, Watsford M. Differences in the kinematics of the baseball swing between hitters of varying skill. Med Sci Sports Exerc. 2011; 43(6): 1050-1054.

10. Szymanski DJ, DeRenne C, Spaniol FJ. Contributing factors for increased bat swing velocity. $J$ Strength Cond Res. 2009; 23(4):1338-1352.

11. Szymanski DJ, Szymanski JM, Schade RL, et al. The relation between anthropometric and physiological variables and bat velocity of high-school baseball players before and after 12 weeks of training. J Strength Cond Res. 2010; 24(11): 2933-2943.

12. Fry AC, Honnold D, Hudy A, et al. Relationships Between Muscular Strength and Batting Performances in Collegiate Baseball Athletes. J Strength Cond Res. 2011;25:S19.

13. Taliep S, Prim S, Gray J. Upper Body Muscle Strength and Batting Performance in Cricket Batsmen. J Strength Cond Res. 2010;24(12):3484-3487.

14. Burke DT, Tran D, Cui D, et al. Significant progression of load on the musculoskeletal system with extremely high loads, with rapid weekly weight gains, using the Anatoly Gravitational System, in a 10-week training period. Open Access J Sports Med. 2013; 4: 211-219.

15. Burke DT, Bell R, Al-Adawi S, et al. Rate of injury and subjective benefits of gravitational wellness weightlifting. Open Access J Sports Med. 2014; 5: 215-221.

16. Burke DT, Burke DP. Redefining the Boundaries of Weightlifting. Sports Med Rehabil J. 2017; 2(2): 1020 . 